\title{
Abstracts from Nippon Eiseigaku Zasshi (Japanese Journal of Hygiene) vol. 68, no. 1
}

\section{Selenium Supplementation Trials for Cancer Prevention and the Subsequent Risk of Type 2 Diabetes Mellitus: Selenium and Vitamin E Cancer Prevention Trial and After}

Nippon Eiseigaku Zasshi, 68, 1-10 (2013)

Hiroshi Koyama ${ }^{1}$, Mutakin $^{2}$, Rizky Abdulah ${ }^{2}$, Chiho Yamazaki ${ }^{1}$, Satomi Kameo ${ }^{1}$

${ }^{1}$ Gunma University Graduate School of Medicine, Department of Public Health

${ }^{2}$ Padjadjaran University Faculty of Pharmacy

The essential trace element selenium has long been considered to exhibit cancer-preventive, antidiabetic and insulin-mimetic properties. However, recent epidemiological studies have indicated that supranutritional selenium intake and high plasma selenium levels are not necessarily preventive against cancer, and are possible risk factors for developing type 2 diabetes mellitus. The results of the SELECT, Selenium and Vitamin E Cancer Prevention Trial, in which it is hypothesized that the supplementations with selenium and/or vitamin $\mathrm{E}$ decrease the prostate cancer incidence among healthy men in the US, showed that the supplementation did not prevent the development of prostate cancer and that the incidence of newly diagnosed type 2 diabetes mellitus increased among the selenium-supplemented participants.

The Nutritional Prevention of Cancer (NPC) trial showed a decreased risk of prostate cancer among participants taking $200 \mu \mathrm{g}$ of selenium daily for 7.7 years. However, the results of the NPC trial also showed an increased risk of type 2 diabetes mellitus in the participants with plasma selenium levels in the top tertile at the start of the study.

Recently, the association of serum selenium with adipocytokines, such as TNF- $\alpha$, VCAM-1, leptin, FABP-4, and MCP-1, has been observed. Selenoprotein $\mathrm{P}$ has been reported to associated with adiponectin, which suggests new roles of selenoprotein $\mathrm{P}$ in cellular energy metabolism, possibly leading to the increased risk of type 2 diabetes mellitus and also the development of cancer. Further studies are required to elucidate the relationship between selenium and adipocytokines and the role of selenoprotein $\mathrm{P}$ in the development of type 2 diabetes mellitus and cancer at high levels of selenium.

\section{Prognostic Prediction of the Functional Capacity and Effectiveness of Functional Improvement Program of the Musculoskeletal System among Users of Preventive Care Service under Long-Term Care Insurance}

Nippon Eiseigaku Zasshi, 68, 11-21 (2013)

Toshimasa Sone ${ }^{1,2}$, Naoki Nakaya ${ }^{3}$, Yasutake Tomata ${ }^{1}$, Jun Aida ${ }^{4}$, Ichiro Okubo $^{5}$, Satoko Ohara ${ }^{6}$, Shuichi Obuchi ${ }^{7}$, Michiko Sugiyama, Seiji Yasumura ${ }^{9}$, Takao Suzuki ${ }^{7,10}$, Ichiro Tsuji ${ }^{1}$

${ }^{1}$ Tohoku University Graduate School of Medicine

${ }^{2}$ Tohoku Fukushi University

${ }^{3}$ Kamakura Women's University

${ }^{4}$ Tohoku University Graduate School of Dentistry

${ }^{5}$ University of Tsukuba Graduate School of Comprehensive Human Sciences

${ }^{6}$ Tokyo Medical and Dental University, Dental Hospital

${ }^{7}$ Tokyo Metropolitan Institute of Gerontology

${ }^{8}$ Kanagawa University of Human Services

${ }^{9}$ Fukushima Medical University

${ }^{10}$ National Institute for Longevity Sciences

Objective: The purpose of this study was to examine the effectiveness of the Functional Improvement Program of the Musculoskeletal System among users of Preventive Care Service under Long-Term Care Insurance.

Methods: A total of 3,073 subjects were analyzed. We used the prediction formula to estimate the predicted value of the Kihon Checklist after 1 year, and calculated the measured value minus the predicted value. The subjects were divided into two groups according to the measured value minus predicted value tertiles: the lowest and middle tertile (good-to-fair measured value) and the highest tertile (poor measured value). We used a multiple logistic regression model to calculate the odds ratio (OR) and $95 \%$ confidence interval (CI) of the good-to-fair measured values of the Kihon Checklist after 1 year, according to the Functional Improvement Program of the Musculoskeletal System.

Results: In potentially dependent elderly, the multivariate adjusted ORs (95 \% CI) of the good-to-fair measured values were 2.4 (1.3-4.4) for those who attended the program eight times or more in a month (vs those who attended it three times or less in a month), 1.3 (1.0-1.8) 
for those who engaged in strength training using machines (vs those who did not train), and $1.4(1.0-1.9)$ for those who engaged in endurance training.

Conclusions: In this study, among potentially dependent elderly, those who attended the program eight times or more in a month and those who engaged in strength training using machines or endurance training showed a significant improvement of their functional capacity.

\section{Factors Associated with Activities of Daily Living (ADL) in Independently Living Elderly Persons in a Community: a Baseline Examination of a Large Scale Cohort Study, Fujiwara-kyo Study}

Nippon Eiseigaku Zasshi, 68, 22-32 (2013)

Masayo Komatsu ${ }^{1,2}$, Satoko Nezu ${ }^{1}$, Kimiko Tomioka ${ }^{1}, \mathrm{Kan}_{\text {Hazaki }}{ }^{3}$, Akihiro Harano ${ }^{4}$, Masayuki Morikawa ${ }^{5}$, Masahiro Takagi ${ }^{2}$, Masahiro Yamada ${ }^{6}$, Yoshitaka Matsumoto ${ }^{7}$, Junko Iwamoto ${ }^{8}$, Rika Ishizuka ${ }^{9}$, Keigo Saeki ${ }^{1}$, Nozomi Okamoto ${ }^{1}$, Norio Kurumatani ${ }^{1}$ ${ }^{1}$ Department of Community Health and Epidemiology,

Nara Medical University School of Medicine

${ }^{2}$ Nara Prefectural Katsuragi Public Health Center

${ }^{3}$ Department of Biomedical Engineering,

Osaka Electro-Communication University

${ }^{4}$ Department of Orthopaedic Surgery, Hanna Chuo Hospital

${ }^{5}$ Sakai City Mental Health Center

${ }^{6}$ Nara Prefectural Koriyama Public Health Center

${ }^{7}$ Nara City Public Health Center, Nara Prefecture

${ }^{8}$ Department of Adult Nursing Science, Yokkaichi Nursing

and Medical Care University

${ }^{9}$ Faculty of Contemporary Human Life Science,

Tezukayama University

Objectives: To investigate factors associated with activities of daily living in independently living elderly persons in a community.

Subjects and Methods: The potential subjects were 4,472 individuals aged 65 years and older who voluntarily participated in a large cohort study, the Fujiwara-kyo study. We used self-administered questionnaires consisting of an activities of daily living (ADL) questionnaire with the Physical Fitness Test established by the Ministry of Education, Culture, Sports, Science and Technology (12 ADL items) to determine the index of higher-level physical independence, demographics, Geriatric Depression Scale, and so on. Mini-mental state examination, measurement of physical fitness, and blood tests were also carried out. A lower ADL level was defined as having a total score of the 12 ADL items (range, 12-36 points) that was below the first quartile of a total score for all the subjects. Factors associated with a low ADL level were examined by multiple logistic regression. Results: A total of 4,198 remained as subjects for analysis. The male, female and 5-year-old groups showed significant differences in the median score of $12 \mathrm{ADL}$ items between any two groups. The highest odds ratio among factors associated with lower ADL level by multiple logistic regression with mutually adjusted independent variables was 4.49 (95\%CI: 2.82-7.17) in the groups of "very sharp pain" or "strong pain" during the last month. Low physical ability, selfawareness of limb weakness, a BMI of over 25, low physical activity, cerebrovascular disorder, depression, low cognitive function, unable "to see normally", unable "to hear someone", "muscle, bone and joint pain" were independently associated with lower ADL level. Conclusion: Multiple factors are associated with lower ADL level assessed on the basis of the 12 ADL items.
Study of the Dietary Preferences and the SocialPsychological Factors that Affect the Dietary Behaviors of High School and University Students

Nippon Eiseigaku Zasshi, 68, 33-45 (2013)

Junichi Kasamaki

Institute of Humanities, Social Sciences and Education, Niigata University

Objectives: This study was conducted to elucidate the correlation among dietary intake, dietary preferences, and social-psychological factors in the youth and to examine the factors that affect such dietary behaviors as snacking, skipping breakfast, and taking a biased nutrition.

Methods: A survey was carried out using a questionnaire with closed questions on multiple items such as dietary behaviors, psychosocial stress, dietary externalization, information and consciousness about health. The survey was conducted on 1,056 high school students and 1,323 university students in Japan.

Results: As a result of the factor analysis among the groups of male/ female and high school/university students, relationships were found between the items of "preferences for snacking" and "snack food intakes" among all these groups. Those who like sweets and snacks tended to snack between lunch and dinner or after dinner by themselves more often than those who do not.

In contrast to men, intermediate correlations were found between the item of "a meal as a diversion" and each of the items of "snack food intake," "preferences for fried foods/sautéed foods/meat dishes," and "preferences for snacking," among women who do not live alone, regardless of their being high school or university students.

The item of "stress over human relationships/academic performance" was shown to have similarly weak correlations with the items of "reasons for skipping breakfast" and "nutrition intake" in the groups of male and female high school students. The less they value nutrition intake, the more they tend to be conscious of stress over human relationships/academic performance.

\section{Determination of Nicotine, Tobacco-Specific Nitrosamine and Mutagenic Activity of Whole Tobacco in Japanese Cigarettes}

Nippon Eiseigaku Zasshi, 68, 46-52 (2013)

Yohei Inaba, Tadamichi Ohkubo, Shigehisa Uchiyama,

Naoki Kunugita

Department of Environmental Health,

National Institute of Public Health

Objective: To measure the nicotine and tobacco-specific nitrosamine (TSNA) contents in whole tobacco from a variety of Japanese domestic cigarette brands and to determine mutagencity.

Methods: The test cigarettes were the top ten best selling cigarette brands in Japan in 2006. The nicotine content in whole tobacco was measured by a modified version of the CORESTA Recommended method. The level of TSNA was measured by a modified version of the Health Canada method. Mutagenicity was assessed using the Salmonella typhimurium strains TA100, TA98 and YG1024 with the metabolic activation system (S9mix) by preincubation assays.

Results: The nicotine content in the test cigarettes averaged $15.7 \pm 1.2 \mathrm{mg} / \mathrm{g}$, and was in the range between 13.7 and $17.2 \mathrm{mg} / \mathrm{g}$. The level of TSNA averaged $1,750 \mathrm{ng} / \mathrm{g}$, and was in the range between 931 and 2,490 ng/g. Mutagenicity was pseudopositive in several samples of the YG1024 tester strain with and without S9mix. 
Conclusion: The cigarettes brands were categorized into four groups (Ultra-low, Low, Medium, and High) based on the nominal nicotine yield figures printed on the cigarette packets. The nicotine content in whole tobacco of the High group was the highest. However, the level of TSNA of the high group was the lowest. The analyses of hazardous chemical compounds in whole tobacco can contribute to the reduction and regulation of the toxicity of tobacco products.

\section{Lead Content of Bones Excavated from Archaeological Sites in Hokkaido}

Nippon Eiseigaku Zasshi, 68, 53-57 (2013)

Jun Yoshinaga $^{1}$, Aya Hisada ${ }^{1}$, Minoru Yoneda ${ }^{2}$, Hajime Ishida ${ }^{3}$

${ }^{1}$ Department of Environmental Studies, University of Tokyo

${ }^{2}$ University Museum, University of Tokyo

${ }^{3}$ Faculty of Medicine, University of Ryukyus

Objectives: The lead content of excavated bone samples from archaeological sites in Hokkaido was measured to obtain insight into the source of human lead contamination known in the historic Japanese Edo era.

Methods: Fifty-seven rib samples excavated from 11 sites of five different eras in Hokkaido were analyzed for lead $(\mathrm{Pb})$, calcium $(\mathrm{Ca})$, and iron $(\mathrm{Fe})$ contents by ICP mass spectrometry and ICP emission spectrometry.

Results: The $\mathrm{Pb} / \mathrm{Ca}$ ratio (mg $\mathrm{Pb} / \mathrm{kg} \mathrm{Ca}$ ) was low (approximately 2.0 ) and constant from the Jomon (approximately $5000 \mathrm{BP}$ ) to the Satsumon (approximately $750 \mathrm{BP}$ ) eras; however, its median increased to 11 in the Modern era. This elevation of $\mathrm{Pb} / \mathrm{Ca}$ ratio in the bone samples from sites of the Modern era was not considered to be due to a greater bone contamination with soil particles because of similar Fe concentrations in the bone samples from this era to those in other eras. This historic trend of $\mathrm{Pb} / \mathrm{Ca}$ ratio was similar to that observed in other parts of Japan. The elevated $\mathrm{Pb} / \mathrm{Ca}$ ratio in the bone samples excavated from sites in the Modern era in other parts of Japan, that is, Edo era, has been ascribed to the usage of face powder containing $\mathrm{Pb}$; however, people inhabiting Hokkaido in those days, the Ainu, were not considered to have the custom of using face powder.

Conclusions: Contamination source(s) other than face powder was postulated in the Modern era of both Hokkaido and other parts of Japan. 